\title{
Correction to: Relating entertainment features in screenplays to movie performance: an empirical investigation
}

\author{
Brianna JeeWon Paulich ${ }^{1}$ • V. Kumar ${ }^{2,3,4,5}$
}

Published online: 23 July 2021

(C) Academy of Marketing Science 2021

\section{Correction to: Journal of the Academy of Marketing Science} https://doi.org/10.1007/s11747-021-00794-y

In the original version of this article, there are typographical errors in Equations 1 and 2. Please see below correction equations:

$$
\begin{aligned}
\ln \left(\text { Box Office Performance }_{i j}\right)= & \beta_{0 j}+\beta_{1 j} \ln \left(\text { Characters }_{i}\right)+\beta_{2 j} \ln \left(\text { Dialogs }_{i}\right)+\beta_{3 j} \ln \left(\text { Scene }_{\text {Pace }_{i}}\right) \\
& +\beta_{4 j} \ln \left(\text { Emotionality }_{i}\right)+\beta_{5 j} \ln \left(\text { Positivity }_{i}\right)+\beta_{6 j} \ln \left(\text { Emotionality }_{i}\right) \ln \left(\text { Characters }_{i}\right) \\
& +\beta_{7 j} \ln \left(\text { Emotionality }_{i}\right) \ln \left(\text { Dialogs }_{i}\right)+\beta_{8 j} \ln \left(\text { Emotionality }_{i}\right) \ln \left(\text { Scene Pace }_{i}\right) \\
& +\beta_{9 j} \ln \left(\text { Positivity }_{i}\right) \ln \left(\text { Characters }_{i}\right)+\beta_{10 j} \ln \left(\text { Positivity }_{i}\right) \ln \left(\text { Dialogs }_{i}\right) \\
& +\beta_{11 j} \ln \left(\text { Positivity }_{i}\right) \ln \left(\text { Scene Pace }_{i}\right)+\sum_{(m=12)}^{2} \beta_{m j} \theta_{i}+\beta_{14 j} \text { Exposure }_{i}+\beta_{15 j} \text { Quality }_{i} \\
& +\sum_{(k=1)}^{K} \gamma_{k j} \ln \left(X_{i k}\right)+\varepsilon_{i j}
\end{aligned}
$$

$\operatorname{Pr}\left(\right.$ Produced $\left._{i}\right)=\Phi\left(\delta_{0}+\delta_{1}\right.$ SPlength $_{i}+\delta_{2}$ SPlength $_{i}^{2}+\delta_{3}$ PriorSPAward $_{i}+\delta_{4}$ PriorSP $_{i}+\delta_{5}$ Book- $\left._{\text {based }}\right)$

The original article has been corrected.

Publisher's note Springer Nature remains neutral with regard to jurisdictional claims in published maps and institutional affiliations.

The online version of the original article can be found at https://doi.org/ 10.1007/s11747-021-00794-y

V. Kumar

vk@isb.edu

Brianna JeeWon Paulich

paulich@usf.edu

1 Muma College of Business, University of South Florida, Tampa, FL, USA

2 Indian School of Business, Hyderabad, India

3 MICA, Ahmedabad, India

4 IIM, Ahmedabad, India

5 WE School, Mumbai, India 